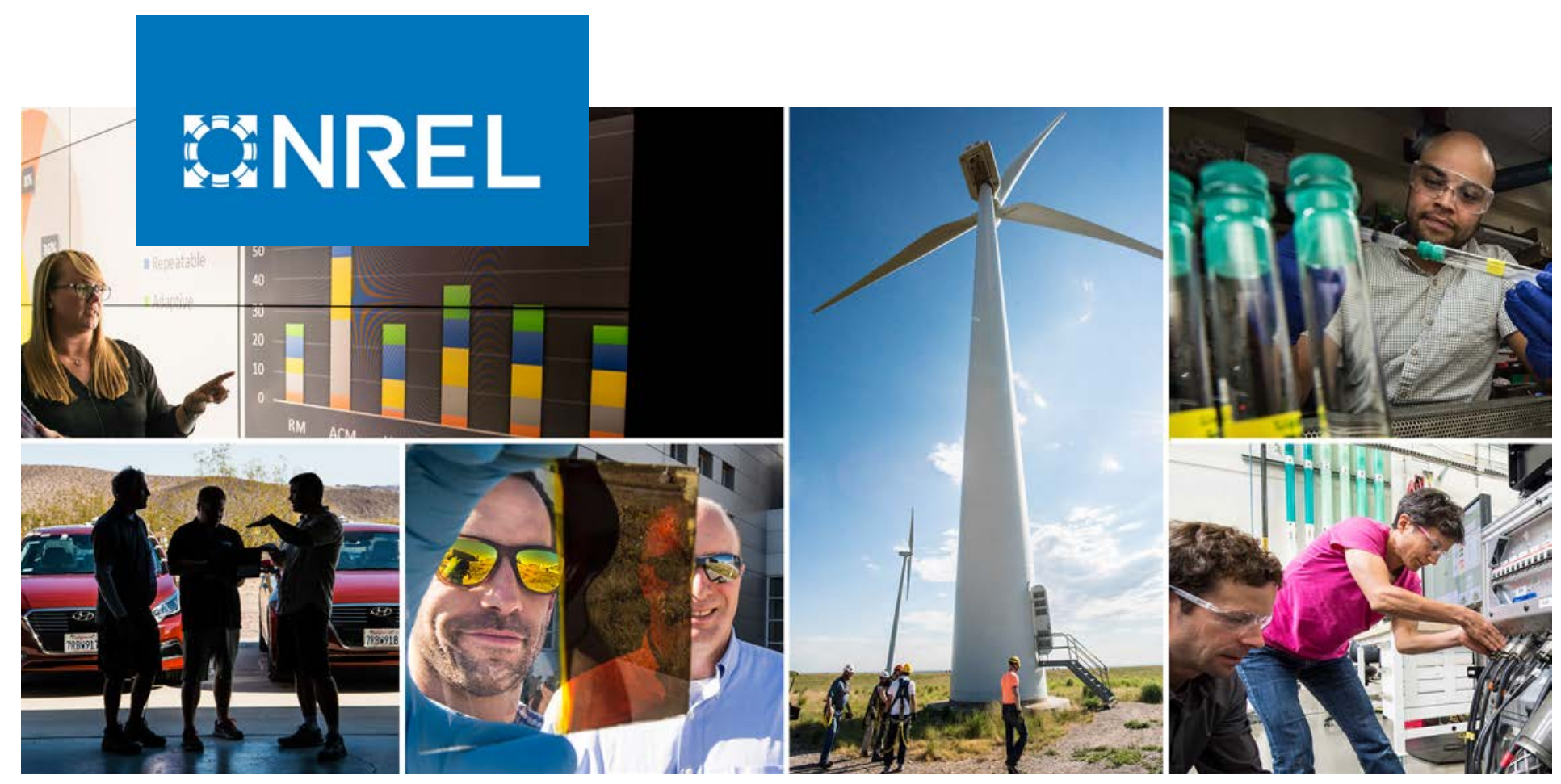

\title{
A Novel and Practical Method to Quantify the Quality of Mobility: The Mobility Energy Productivity Metric
}

\section{Preprint}

Yi Hou, Venu Garikapati, Ambarish Nag, Stanley Young, and Tom Grushka

National Renewable Energy Laboratory

Presented at Transportation Research Board (TRB) 98 ${ }^{\text {th }}$ Annual Meeting Washington, $D C$ January 13-17, 2019

NREL is a national laboratory of the U.S. Department of Energy Office of Energy Efficiency \& Renewable Energy

Operated by the Alliance for Sustainable Energy, LLC

This report is available at no cost from the National Renewable Energy Laboratory (NREL) at www.nrel.gov/publications.

\section{Conference Paper}

NREL/CP-5400-72889

February 2020 


\title{
ENREL
}

\section{A Novel and Practical Method to Quantify the Quality of Mobility: The Mobility Energy Productivity Metric}

\section{Preprint}

\author{
Yi Hou, Venu Garikapati, Ambarish Nag, Stanley Young, \\ and Tom Grushka \\ National Renewable Energy Laboratory
}

\section{Suggested Citation}

Hou, Yi, Venu Garikapati, Ambarish Nag, Stanley Young, and Tom Grushka. 2020. A Novel and Practical Method to Quantify the Quality of Mobility: The Mobility Energy Productivity Metric: Preprint. Golden, CO: National Renewable Energy Laboratory. NREL/ CP-5400-72889. https://www.nrel.gov/docs/fy20osti/72889.pdf.

NREL is a national laboratory of the U.S. Department of Energy Office of Energy Efficiency \& Renewable Energy Operated by the Alliance for Sustainable Energy, LLC

This report is available at no cost from the National Renewable Energy Laboratory (NREL) at www.nrel.gov/publications.

Contract No. DE-AC36-08GO28308
Conference Paper NREL/CP-5400-72889 February 2020

National Renewable Energy Laboratory 15013 Denver West Parkway Golden, CO 80401

303-275-3000 • www.nrel.gov 


\section{NOTICE}

This work was authored by the National Renewable Energy Laboratory, operated by Alliance for Sustainable Energy, LLC, for the U.S. Department of Energy (DOE) under Contract No. DE-AC36-08GO28308. Funding provided by the U.S. Department of Energy Office of Energy Efficiency and Renewable Energy Vehicle Technologies Office. The views expressed herein do not necessarily represent the views of the DOE or the U.S. Government. The U.S. Government retains and the publisher, by accepting the article for publication, acknowledges that the U.S. Government retains a nonexclusive, paid-up, irrevocable, worldwide license to publish or reproduce the published form of this work, or allow others to do so, for U.S. Government purposes.

This report is available at no cost from the National Renewable Energy Laboratory (NREL) at www.nrel.gov/publications.

U.S. Department of Energy (DOE) reports produced after 1991 and a growing number of pre-1991 documents are available free via www.OSTI.gov.

Cover Photos by Dennis Schroeder: (clockwise, left to right) NREL 51934, NREL 45897, NREL 42160, NREL 45891, NREL 48097, NREL 46526.

NREL prints on paper that contains recycled content. 


\title{
A NOVEL AND PRACTICAL METHOD TO QUANTIFY THE QUALITY OF MOBILITY: THE MOBILITY ENERGY PRODUCTIVITY METRIC
}

\author{
Yi Hou, Ph.D. \\ National Renewable Energy Laboratory \\ 15013 Denver West Parkway, Golden, Colorado 80401 \\ Tel: 303-384-7525 \\ Email: yi.hou@nrel.gov \\ Venu Garikapati, Ph.D. \\ National Renewable Energy Laboratory \\ 15013 Denver West Parkway, Golden, Colorado 80401 \\ Tel: $303-275-4784$ \\ Email: venu.garikapati@,nrel.gov \\ Ambarish Nag, Ph.D. \\ National Renewable Energy Laboratory \\ 15013 Denver West Parkway, Golden, Colorado 80401 \\ Tel: 303-275-3754 \\ Email: ambarish.nag@nrel.gov \\ Stanley E. Young, Ph.D. \\ National Renewable Energy Laboratory \\ 15013 Denver West Parkway, Golden, Colorado 80401 \\ Tel: 303-275-3283 \\ Email: stanley.young@,nrel.gov \\ Tom Grushka \\ National Renewable Energy Laboratory \\ 15013 Denver West Parkway, Golden, Colorado 80401 \\ Tel: 303-275-3645 \\ Email: tom.grushka@nrel.gov
}

Submitted for consideration for presentation and publication at TRB Annual Meeting

Washington, D.C. January 2019

Words in Text: 7058

Number of Tables: 2

Number of Figures: 6

Total word count: 7558

Revised: November 15, 2018 


\begin{abstract}
Recent technology innovations are enabling fundamental improvements in mobility systems, including options for new travel modes, methods, and opportunities to connect people with goods, services, and employment. A desire to quantify and compare both existing and emerging transportation options motivated development of the mobility energy productivity (MEP) metric described herein. The MEP metric fundamentally measures the potential of a city's transportation system to connect a person to a variety of services and activities that define a high-quality of life, relative to the convenience, cost and energy needed to provide these connections. Fundamentally derived from accessibility theory, the MEP advances practice by using readily available travel time data (either from web-based application programming interfaces (APIs) or outputs from an urban transportation model) combined with established parameters that reflect the energy intensity and cost of various travel modes, and relative frequency of activity engagement. The construction of the MEP metric allows for aggregation and disaggregation to the appropriate spatial, modal, and trip purpose resolution, as analysis needs dictate. The MEP could be used to compare alternative futures related to technology, infrastructure investment, or policy, providing a much-needed tool for planners, researchers, and analysts.
\end{abstract}

Keywords: mobility, accessibility, isochrones, energy, productivity 


\section{INTRODUCTION}

Mobility is one of the fundamental aspects of human behavior, but has proven elusive to quantify objectively. The ability for people to get where they need to go expending a minimum amount of time, cost, and energy is a critical factor to quality of life. The Department of Energy's (DOE's) Energy Efficient Mobility Systems program is examining the potential mobility and energy impacts of multiple new technologies encompassing vehicle automation, connectivity, electrification, and sharing (collectively referred to as ACES). ACES has paved the way to mobility-as-a service (MaaS) with offerings such as ride-sharing, bike-sharing, car-sharing, and other constantly emerging business models. The influences of ACES technologies are beginning to make shifts in the United States' long-standing automotive culture $(1,2,3)$ and are anticipated to drastically change the nature of surface mobility in decades to come, particularly in urban areas. In this new era, older roadway-infrastructure methods of measuring transportation system efficiency fall short. Measures such as vehicle miles traveled, miles per gallon (MPG), travel time, and congestion are uni-dimensional and fail to capture true mobility.

Is an individual who commutes 60 miles to work more "mobile" than an urban dweller that has given up vehicle ownership, choosing transit, car-share, ride-shares, and other mobility offerings? To answer this question, one needs to have a clear understanding of the quality of mobility that home/work locations of each of these individuals provide. The DOE's Energy Efficient Mobility Systems program has the objective of identifying and developing system-level transportation technologies and innovations that enable an increase in mobility while reducing energy inefficiency. While there are a variety of metrics to quantify energy efficiency (MPG, EnergyStar rating etc.), there are sparse metrics for quantifying the quality of mobility. These concerns sparked the necessity to define an appropriate quantitative lens from which the connectivity benefits afforded people by traditional modes as well as new mobility technologies could be viewed holistically with respect to travel time, energy, and affordability. This concept and the resulting metric work that has evolved have come to be called the Mobility Energy Productivity metric - or simply the MEP. The term "productivity" in the context of this work is defined as efficiency of our transportation system to produce useful outputs. Mobility is defined as the quality of our transportation system to connect people to goods, services, and employment that define a high quality of life. The MEP measures the efficiency of that system, relative to travel time, cost, and energy. It should be noted at the outset that the MEP is a measure of the potential field that enumerates the richness of opportunities one can reach from a given location (using a variety of modes) and weights the opportunity measure along time, cost, and energy dimensions. The metric proposed herein uses weighting parameters derived from published national sources; however, the authors note that the MEP can be fine-tuned to a region or population sub-group based on known data sources such as regional surveys and other sources that typically support a regional travel demand model.

Cities are struggling to find an objective, data-driven analysis framework to assess the tradeoffs in investment decisions with respect to transportation, be it traditional road infrastructure or policy and pricing related to new mobility businesses (such as Uber and Lyft), parking pricing and availability, and/or re-use of curb-fronts for shared mobility services. The MEP provides a lens through which impacts of such competing investments over a region can be objectively compared and quantified. The MEP can also be utilized to understand impacts of technological improvements (increase in vehicle efficiency, decrease in value of travel time through vehicle automation, etc.) on the mobility of a region. 
The remainder of the paper is organized as follows. The next section provides a review of literature on relevant accessibility and mobility metrics. The third section presents the framework and fundamental methodology of the MEP. The fourth section presents the data sources used to compute the MEP metric. The fifth section presents results of the MEP computation for Columbus, Ohio. The sixth section details scenario analyses carried out with the MEP metric. Discussion and conclusions are presented in the seventh and final section.

\section{LITERATURE REVIEW}

Since the seminal works of Ravenstein (4), human mobility has been an active area of research in social and geographical sciences. All the quantitative studies in this area have established that a close relationship exists between mobility, distance, and accessibility. Travel patterns of individuals exhibit high levels of regularity (for example, weekday travel pattern of a worker involves more or less the same set of activities across different days of week) and tend to be hindered by geographical distance (5). Such movements are biased towards areas that are more accessible by road infrastructure and transportation systems. Several researchers $(6,7,8,9)$ have summarized the key elements of accessibility to be (1) the spatial distribution of opportunities, (2) mobility provided by the road infrastructure and transportation system, (3) temporal constraints of individuals and activities, and (4) individual characteristics of people. The concepts of mobility and accessibility are inextricably linked and are often used interchangeably.

Mobility and accessibility measures in the current literature can be broadly classified into five major categories, namely infrastructure-based, location-based, person-based, utility-based, and activity-based measures $(6,7,8)$. It should be emphasized that these categories are not mutually exclusive but there exists some degree of overlap between them. Infrastructure-based measures depict the observed or simulated performance or service level of transportation infrastructure, e.g., congestion level and average travel speed on the road network $(7,10)$. The advantage of infrastructure-based measures is that they can be easily computed and communicated to even nontechnical decision makers (7). Their major shortcoming is the lack of incorporation of land use, temporal and individual components of accessibility (7).

Location-based measures analyze the degree of accessibility at locations, typically on a macrolevel (10) to spatially distributed opportunities. Location-based measures have proved to be very useful in understanding both regional and local accessibility (7). There are three kinds of locationbased measures:

- Distance-based measures: These location-based measures involve the relative accessibility between any two locations in terms of travel times, distances, or costs (7).

- Isochrone-based measures: The isochrone-based measures are cumulative opportunity measures that involve the construction of concentric areas of various cost budgets (6). Examples of this metric have been provided by Wachs and Kumagai (11), and Vickerman (12), and Silva and Pinho (13).

- Potential accessibility/ Gravity-based measures: The gravity-based measures are similar to the isochrone-based measures with the modification that the opportunities are weighted by the travel cost of reaching them $(6,14)$.

Two diverging viewpoints on gravity-based measures have emerged from the classical quantitative studies of accessibility/mobility metrics (15). One viewpoint is that mobility is directly deterred by the costs (time and energy) associated with physical distance $(16,17,18)$. The other viewpoint 
involves the argument that there is no direct relation between mobility and distance, and that distance is a substitute for the effect of intervening opportunities (19). The first of the above two viewpoints seems to have been preferred by practitioners because of computational ease (20), in spite of the fact that several statistical studies have shown that the concept of intervening opportunities is better at explaining a broad range of mobility data $(21,22,23,24,25)$. These studies establish that, in the context of mobility quantification, the number of opportunities within a specified distance is more informative than the physical distance itself or the time and energy costs associated with the physical distance. Stouffer's (19) hypothesis about intervening opportunities was validated using migration data by Miller (21) and Haynes et al. (22). Wadycki (23) undertook a study based on interstate migration flows in the continental United States which established that Stouffer's theory of intervening opportunities was consistently valid at different levels of aggregation. Cheung and Black (24) showed that in a zonal-specific spatial distribution context, the Census journey to work data for Canberra, Australia, favored the interveningopportunities model over a gravity-based approach.

Person-based measures analyze the degree of accessibility of individuals with certain monetary and temporal budgets to spatially distributed opportunities in terms such as the number of jobs that an individual can participate in at a given time. However, they are difficult to compute using available land use and transportation system data due to sparsity or unavailability of survey and time/cost budget data (7).

Another commonly used accessibility measure is the Expected Maximum Utility (EMU) or the logsum measure (6), which is an output from the logit destination choice model. The logsum measure represents the cutting edge in measuring accessibility. Its power stems from the ability to recognize differences in attractiveness of opportunities to different segments of the population. The accessibility measured using this metric can differ for individuals at the same location who belong to different socio-economic backgrounds. However, the dependence of this measure on disaggregate travel behavior makes data availability difficult, thereby limiting the applicability of this measure. Applications of this class of accessibility measures have been undertaken by BenAkiva and Lerman (26) and by Dong and co-workers $(27,28)$.

Activity-based measures extend beyond the trip-making behavior of individuals and instead focus on the benefits associated with the activities that an individual participates in throughout the day (e.g., shopping, working, etc.) (7). These metrics incorporate traditionally hard-to-model situations like working from home, etc., and thus provide, at least theoretically, a more complete picture of accessibility. These measures are thus better than utility-based measures; however, they have some major disadvantages including being data-hungry. In addition to the above measures of mobility/accessibility, a novel framework has been developed by Simini et al. (29) in which a stochastic process is used to capture local mobility decisions that enable the analytic derivation of commuting and mobility fluxes that require input information on population distribution.

In addition to measures found in academic literature, similar metrics have been developed by non-academic, government, and private entities. Often these methodologies, particularly those from for-profit companies, are not completely disclosed. Notable among these are the Walk Score $^{\circledR}$, Transit Score, and Bike Score metrics (30). More recently, Transit Screen ${ }^{\circledR}$ proposed the MobilityScore (31), which considers all possible transportation options from public transport to car-sharing, bike-sharing, and hailed ride-sharing services to yield an indicative metric of a 
region's mobility. For all the aforementioned metrics, each physical location is assigned a score in the range 0 to 100 where 0 implies complete dependence on automobiles and 100 implies easy and quick reachability by walking/biking/transit or availability of multiple transportation choices. While the MobilityScore considers a greater range of modes than Walk Score ${ }^{\circledR}$ (or similar metrics), it does not take into consideration the richness of opportunities that can be accessed using each of the modes at a given location.

In parallel, several researchers have studied urban accessibility from the perspective of energy consumption. Rendall et al. (32) has introduced the concept of active modal accessibility, which is defined as the proportion of activities that can be reached by active modes (walking, cycling, and public transport) alone, to quantify the transportation energy footprint of an area. Inturri et al. (33) confirmed a significant statistical correlation between the accessibility index and the transport energy dependency index of a region. Saunders et al. (34) proposed a method in which the transportation energy used per week by each resident is estimated based on the trip length from each resident's home to each specific activity, the number of trips per week for each activity, and the energy consumption rate of each transportation mode. In related work, Karathodorou et al. (35) evaluated the impact of urban density on transportation fuel demand.

A comprehensive dialogue on the accessibility- and energy-based transportation efficiency metrics can be found in a literature review supplement (36). From a thorough review of the literature on this topic, two drawbacks emerge in current research. The academic literature on transportation efficiency metrics is rich in theory, but oftentimes is limited by data availability and computational burden for widespread application. In contrast, popular industry metrics that have become readily available are mode specific and proprietary, limiting their ability to comprehensively represent a region's mobility. The literature review has revealed the necessity for a comprehensive (including all modes), integrated (including accessibility and energy efficiency of travel), open source (available free of cost), and data agnostic (can make use of readily available data sources) metric that can be applied at any geographical scale to quantify the quality of mobility.

\section{METHODOLOGY}

The framework for developing the MEP metric was established based on the following properties:

- The metric should reflect the relative efficiency (in time, cost, and energy) to access a variety of goods, services, and employment (in other words, intervening opportunities) consistent with most accessibility theory approaches.

- The metric should be able to be applicable to any mode, both existing and yet to be invented, as well as across modes.

- Fundamental modal measurements should include travel time, cost, and energy.

- The measure should be grounded in established research, yet be practical to implement and supported by available data (the latter being where historical accessibility/mobility measures have fallen short).

- The measure should be spatially scalable. At its base, the measure defines the quality of mobility at a place, and then the measure can be spatially aggregated to any level (neighborhood, district, city, region, state, etc.).

- The metric should be able to compare/contrast: (1) different locations such as two cities, (2) disparate planning approaches or infrastructure investments such as a roadway 
expansion versus better land use planning, and (3) impacts of different technologies such as adoption of electric vehicles versus automated vehicles.

- The metric results should be able to quantify the mobility energy productivity in a single number for an entire city, while having the ability to disaggregate across space, mode, or activity to better observe how a new mobility option will impact various aspects of an urban area.

The MEP metric adopts the characteristics of isochrone-based metrics and the concept of intervening opportunities. The methodology presented in this section builds on the strengths of accessibility-related research carried out by Owen and Levinson (37). While most of the existing metrics exclusively focus on a single mode (car, transit, etc.), a single weighting factor (for example, travel time), or a single opportunity type (for example, jobs), the MEP accounts for multiple weighting factors (energy efficiency, affordability, and travel time), multiple modes, and multiple opportunity types.

The MEP methodology can be implemented at any appropriate spatial resolution for which data are available (such as Census Block Group, Census Tract etc.,). The examples herein use a 1-sq. $\mathrm{km}$ pixel resolution to balance computational burden with geographic resolution, and consider four transportation modes: driving, transit, biking, and walking. Broadly speaking, the MEP quantifies opportunity space (the aggregated number of opportunities) that can be accessed from a location, weighted by travel time, energy efficiency, and cost of modes that provide access to the opportunities. Note that any of these dimensions can be omitted for insight. For example, omitting the energy component may yield results more consistent with user perception of mobility (without energy/sustainability concerns). The MEP calculation presented herein uses Columbus, Ohio, as an example. The study area is divided into $1-\mathrm{km} \times 1-\mathrm{km}$ square pixels, as shown in FIGURE 1 . MEP is evaluated at the centroid of each $1 \mathrm{~km} \times 1 \mathrm{~km}$ pixel. 


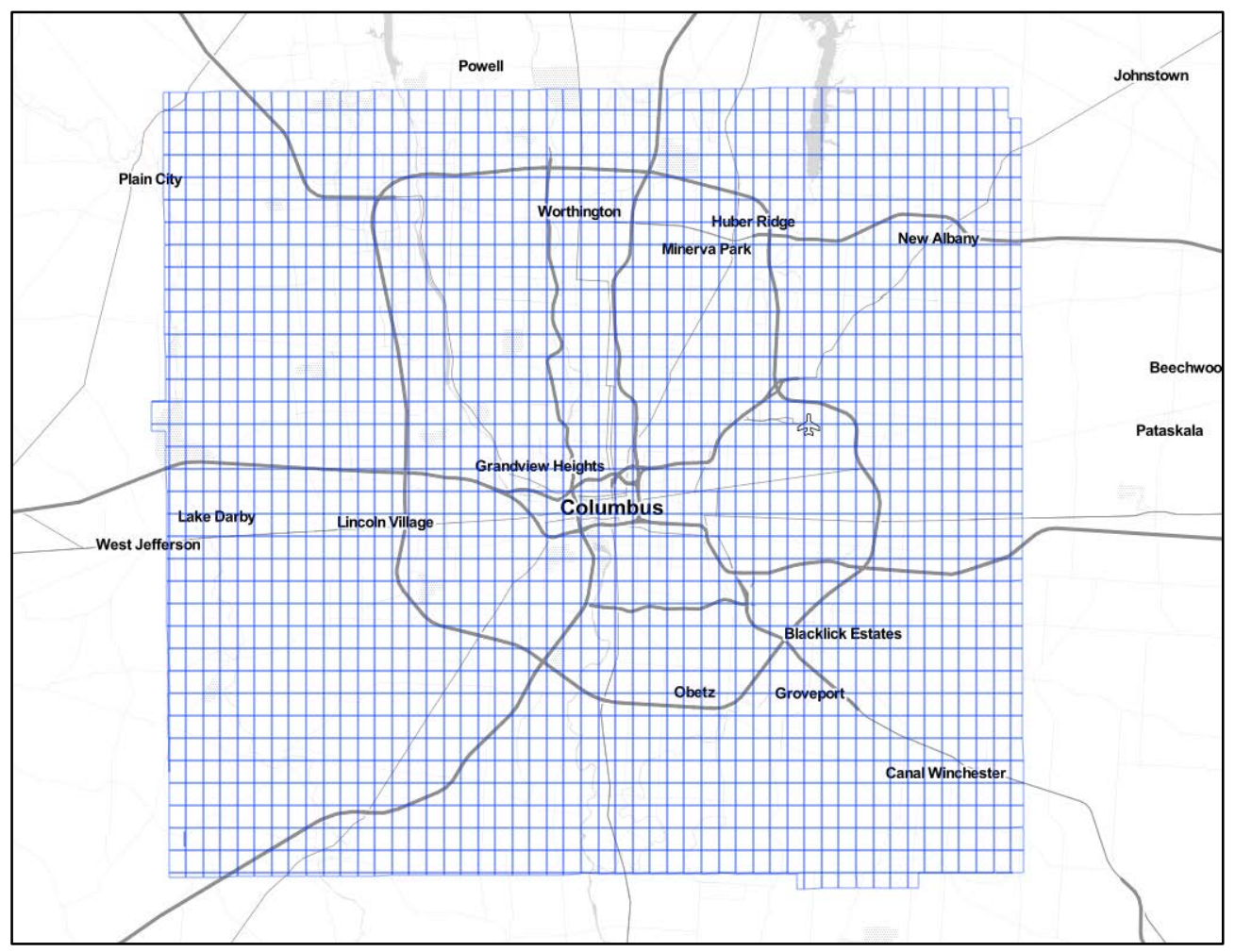

FIGURE 1 The study area of Columbus, $\mathrm{OH}$.

\section{Cumulative Opportunities}

The MEP calculation starts with the computation of a cumulative opportunity measure $(11,12)$ by counting the number of opportunities that can be reached within a certain travel time threshold. To do so, it is necessary to construct the area reachable from a given location within a given amount of travel time for each transportation mode. These areas, termed "isochrones," are strictly defined as polygons formed by a line drawn on a map connecting all the furthest points a traveler can reach from the same origin within a set amount of travel time. The cumulative opportunity measures (for each mode and activity type) are calculated for each square kilometer pixel for different travel time thresholds (see equation 1). The cut-offs for travel time thresholds are user defined and can be as aggregated as an hour or as granular as a minute. Thresholds of 10, 20, 30, and 40 minutes are used for the Columbus, Ohio, implementation of the MEP methodology.

$$
o_{i k t}=\sum_{j} o_{i j k t} \cdot \frac{N^{*}}{N_{j}} \cdot \frac{f_{j}}{\sum_{j} f_{j}}
$$

where

$o_{i j k t} \quad$ is the number of opportunities of activity $j$ that can be accessed by mode $k$ within the travel time threshold $t$ from the $i$ th pixel

$N^{*} \quad$ is the total number of benchmark opportunities across multiple cities (for example, the number of meal opportunities)

$N_{j} \quad$ is the total number of opportunities of activity $j$ (for example, number of shopping opportunities)

$f_{j} \quad$ is the frequency that people access opportunities of activity $j$ 
$o_{i k t} \quad$ is the number of benchmark opportunities that can be accessed by mode $k$ within the travel time threshold $t$ from the $i$ th pixel. For the Columbus, Ohio, example presented in the following section, meal opportunities are chosen as the benchmark opportunity.

The total number of different types of opportunities that can be accessed within a certain amount of time varies in magnitude. For example, there might be thousands of work opportunities accessible from a given location, but only tens of grocery stores or shopping opportunities. Therefore, all opportunities are translated to an equivalent benchmark opportunity measure using a factor $\left(\frac{N^{*}}{N_{j}}\right)$. This proportioning factor is derived based on data from multiple cities. For example, if across multiple cities there are 34,000 meal/restaurant opportunities and 200,000 shopping opportunities, taking meal as the reference category, the $\frac{N^{*}}{N_{j}}$ factor for shopping would be 0.17 . This factor remains constant for application of the MEP metric in any city whereas the raw opportunities measure $o_{i j k t}$ will vary from city to city.

Similarly, it should be noted that people access different types of opportunities at varying frequencies. For example, work is a more regular activity in which people participate on a day-today basis, compared with recreational activities in which people participate less frequently (say on a weekly basis). To accommodate this, the opportunity measure calculated in the previous step is further proportioned using a frequency of activity engagement factor $\left(\frac{f_{j}}{\sum_{j} f_{j}}\right)$.

\section{Modal Weighting Factor}

One of the key facets of MEP that sets it apart from existing metrics is accounting for multiple factors, including modal availability, convenience (travel time), sustainability (energy efficiency), and affordability (cost) in accessing opportunities. More weight should be assigned to opportunities that can be accessed with more available, sustainable, and affordable modes in less travel time, and vice versa. Along this line of thought, the concept of a modal weighting factor is introduced as

$$
M_{i k t}=\alpha e_{k}+\beta t+\sigma c_{k}
$$

where

$M_{i k t}$ is the modal weighting factor for opportunities accessed by mode $k$ with travel time $t$ from location $i$

$e_{k} \quad$ is the energy intensity ( $\mathrm{kWh}$ per passenger-mile) of mode $k$

$t \quad$ is travel time

$c_{k} \quad$ is the cost (dollar per passenger-mile) of using transportation mode $k$ $\alpha, \beta$, and $\sigma$ are weighing factors.

$\beta$ is a weighting factor set to be -0.08 in this study, following Owen and Levinson (37). Both $\alpha$ and $\sigma$ are set to be -0.5 based on the authors' judgement. It is not the intent of the authors to determine or advocate weighting factors through this research. Rather, the weighting factors should be determined and plugged in for specific use cases by researchers utilizing the metric. 


\section{Energy, Cost, and Time Weighted Mobility}

A comprehensive metric to quantify the quality of mobility is then calculated by weighting the cumulative opportunities using a negative exponential function applied on the modal weighting factor. The final equation for computing MEP for a location $i$ is written as:

$$
\mathrm{MEP}_{i}=\sum_{k} \sum_{t}\left(o_{i k t}-o_{i k(t-10)}\right) \cdot e^{M_{i k t}}
$$

Using this methodology, the MEP score can be computed for any geographical entity of choice (a land parcel, census block group, or a census tract). The overall MEP for a city can be calculated by taking the population proportion weighted summation of MEP across tracts or block groups (or any other geographical resolution of choice) in a city. Thus, if most populated regions in a city have high MEP scores, the city-level MEP will be high.

\section{DATA SOURCES}

The primary inputs required to compute the MEP for any given location are the: 1) Isochrones (or travel time information from which isochrones can be constructed) for each mode, 2) land use and employment data, 3) energy intensity and monetary cost of various modes, 4) frequency of activity engagement, and 5) population density. This section describes the data inputs required for calculating MEP. Modes are limited to walking, biking, driving, and transit in this paper (efforts are underway to include additional modes). Data availability and practical implementation of the MEP methodology drove selection of the framework and algorithm.

\section{Isochrones}

For the initial demonstration of MEP computation, isochrones of driving, biking, walking, and transit were queried through Mapzen (38), an open mapping platform. The isochrone service is powered by road network data from OpenStreetMap (39) and public transit data from Transitland (40). When constructing isochrones for transit modes, wait times, transfer times and walk (to destination) times should also be considered to provide a realistic representation of the distance that can be covered using transit. Note that transit travel times can typically be obtained from the General Transit Feed Specification (GTFS) data (41). FIGURE 2 shows an example of isochrones of 10-, 20-, 30-, and 40-minute travel time by biking in Columbus, Ohio. 


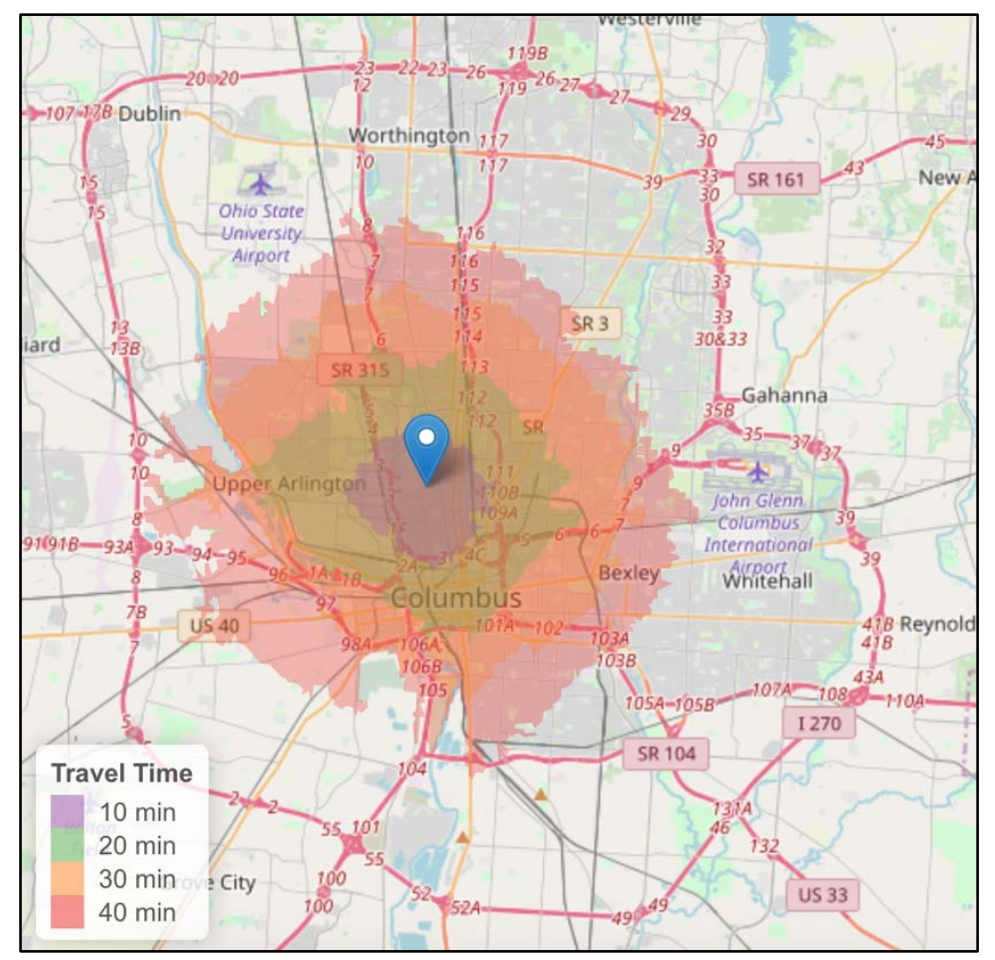

FIGURE 2 Isochrones of 10-, 20-, 30-, and 40-minute travel time by biking.

\section{Land Use and Employment}

Land use and employment information are required to calculate cumulative opportunities $\left(o_{i j k t}\right)$ accessible within the travel time isochrones. Land use data provide the quantity and variety of activity engagement opportunities that can be accessed from a given location. Land use data can be obtained from third-party data providers such as $\operatorname{CoStar}^{\mathrm{TM}}$ (42), Google Places (43), or FourSquare (44) as well as from city planning organizations. For this study, land use data were acquired from CoStar (depicted in FIGURE 3). CoStar provides land use information in the form of data for all physical structures, including building type, location, number of stories, land area size, and purpose. While CoStar provides data for opportunities of various types, it does not provide employment data. Employment data are distilled from the workplace area characteristics data of the Longitudinal Household Employer Dynamics (LEHD) Origin-Destination Employment Statistics of the U.S. Census at the Census block level (45).

CoStar building-type purposes include fast food/restaurant/bar, health care, religious facility/school/day care, retail, and sports and entertainment. The number of work opportunities was calculated by multiplying the employee density with land area size and number of stories of employment attractions, including office, shopping center, industrial, government, and education as queried from the Workplace Area Characteristics data of the LEHD Origin-Destination Employment Statistics of the U.S. Census at the Census block level. 


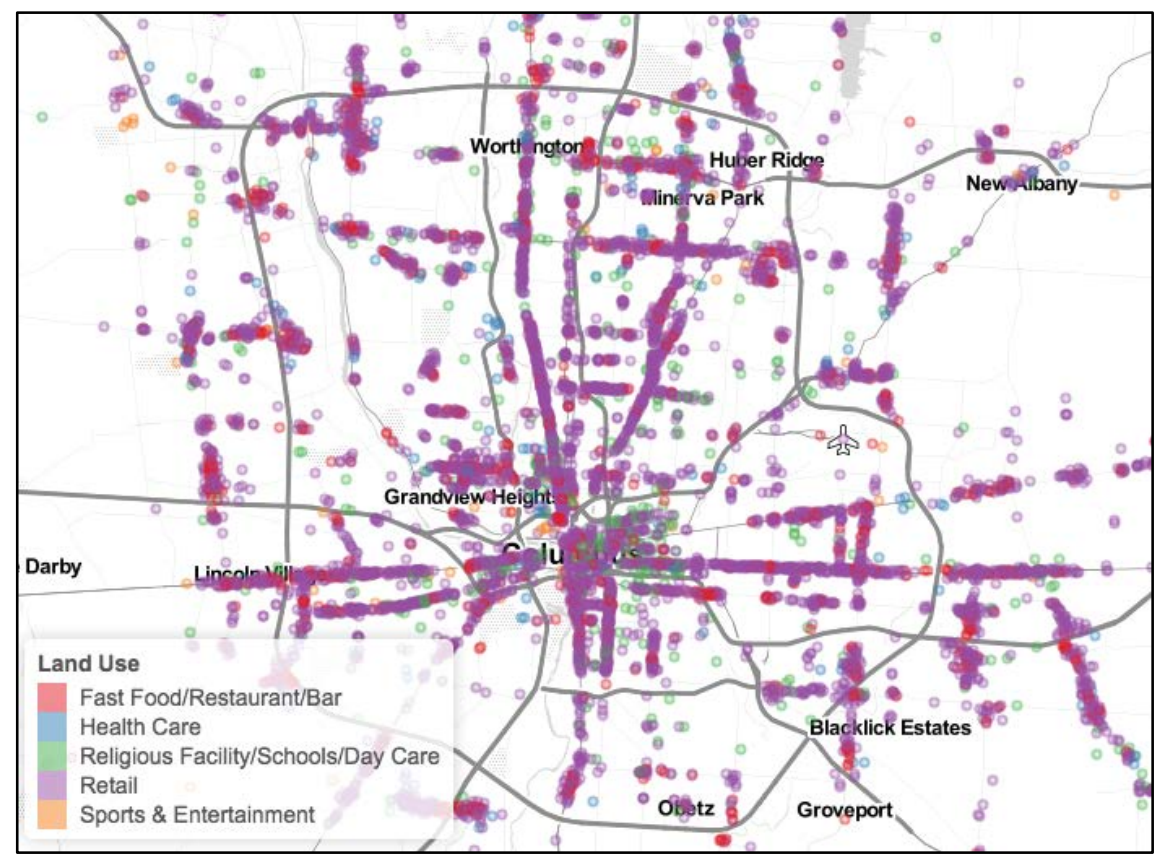

FIGURE 3 Land use data from CoStar.

\section{Energy Use and Monetary Cost Data}

In the MEP calculation procedure, opportunities are weighted by the energy intensity and cost of various (available) travel modes. To account for energy use in accessing opportunities, information regarding kilowatt-hours consumed per passenger-mile of driving and transit is obtained from Transportation Energy Data Book (46). The energy intensities for biking and walking are considered to be zero since biking and walking do not consume any fossil fuel energy. In addition, the capital and operational costs of using each transportation mode are also considered in the MEP calculation. The costs of walking and biking are assumed to be zero dollars per passenger-mile since the costs are minimal when compared with other modes. The costs of transit, and driving are acquired from Federal Transit Administration (47), American Automobile Association (48) respectively. Information regarding energy use and monetary cost for various modes used in MEP calculation are provided in TABLE 1.

\section{TABLE 1 Energy Use per Passenger-Mile by Different Transportation Modes}

\begin{tabular}{|l|c|c|c|c|}
\hline $\begin{array}{l}\text { Transportation } \\
\text { Mode }\end{array}$ & $\begin{array}{c}\text { kWh per } \\
\text { Passenger-Mile }\end{array}$ & $\begin{array}{c}\text { Energy Data } \\
\text { Source }\end{array}$ & $\begin{array}{c}\text { Dollar per } \\
\text { Passenger- } \\
\text { Mile }\end{array}$ & $\begin{array}{c}\text { Cost Data } \\
\text { Source }\end{array}$ \\
\hline Biking & 0 & N/A & 0 & N/A \\
\hline Walking & 0 & N/A & 0 & N/A \\
\hline Transit & 0.650 & $\begin{array}{c}\text { Transportation } \\
\text { Energy Data } \\
\text { Book (46) }\end{array}$ & 1.05 & $\begin{array}{c}\text { Federal } \\
\text { Transit } \\
\text { Administration } \\
\text { (47) }\end{array}$ \\
\hline Driving & 0.900 & $\begin{array}{c}\text { Transportation } \\
\text { Energy Data } \\
\text { Book (46) }\end{array}$ & 0.48 & $\begin{array}{c}\text { American } \\
\text { Automobile } \\
\text { Association } \\
\text { (48) }\end{array}$ \\
\hline
\end{tabular}




\section{Frequency and Categorization of Activity Engagement}

People engage in different activities at varying frequencies, and the MEP calculation takes this into account. Trip frequencies $\left(f_{j}\right.$ in Equation 1$)$ and corresponding activity categorizations were obtained from the 2017 National Household Travel Survey (NHTS) data (49). The NHTS activity types correspond one-to-one with the CoStar land use categories, as shown in TABLE 2.

TABLE 2 Trip Frequency of Different Activities

\begin{tabular}{|l|c|c|}
\hline CoStar Land Use & $\begin{array}{c}\text { NHTS Activity } \\
\text { Category }\end{array}$ & NHTS Trip Frequency \\
\hline $\begin{array}{l}\text { Religious } \\
\text { Facility/Schools/Day } \\
\text { Care }\end{array}$ & $\begin{array}{c}\text { School/Daycare/ } \\
\text { Religious Activity }\end{array}$ & $3.1 \%$ \\
\hline Health Care & $\begin{array}{c}\text { Medical/ } \\
\text { Dental services }\end{array}$ & $1.5 \%$ \\
\hline Retail & $\begin{array}{c}\text { Shopping/ } \\
\text { Errands }\end{array}$ & $19.5 \%$ \\
\hline Sports \& Entertainment & $\begin{array}{c}\text { Social/ } \\
\text { Recreational }\end{array}$ & $8.4 \%$ \\
\hline $\begin{array}{l}\text { Fast } \\
\text { food/Restaurant/Bar }\end{array}$ & Meals & $6.7 \%$ \\
\hline Jobs & Work & $16.6 \%$ \\
\hline
\end{tabular}

\section{Population}

Population estimates were obtained from the U.S. Census 2016 American Community Survey (50) 5 -year estimates at the Census block group level. The population at each location (a 1-km by 1-km pixel in these examples) was calculated by multiplying the population estimate in each block group intersecting the grid cell by the proportion of its area within the grid cell. These data are used to aggregate MEP from all the pixels in a city to an overall city-level metric.

\section{RESULTS}

Using the methodology and data sources described above, the MEP was calculated for the Columbus, Ohio, metropolitan region. Figures $4 \mathrm{a}-4 \mathrm{c}$ present the graphical depiction of MEP for driving, all modes except driving, and the combined MEP, respectively. Note that no population weighting or aggregation is depicted in these graphics. 


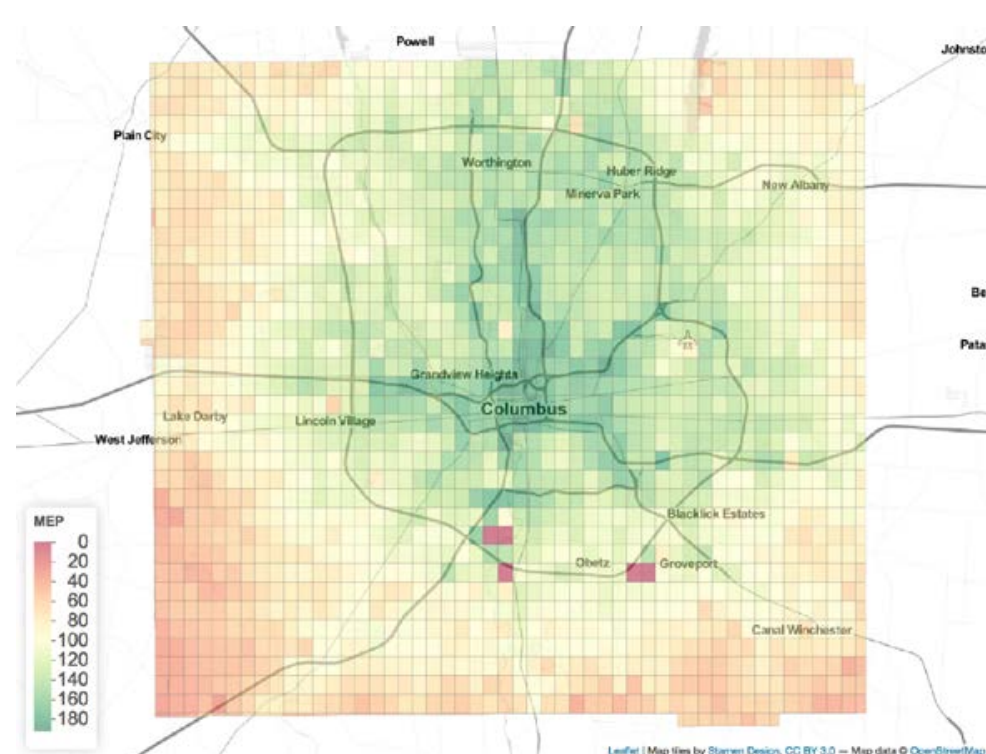

a)

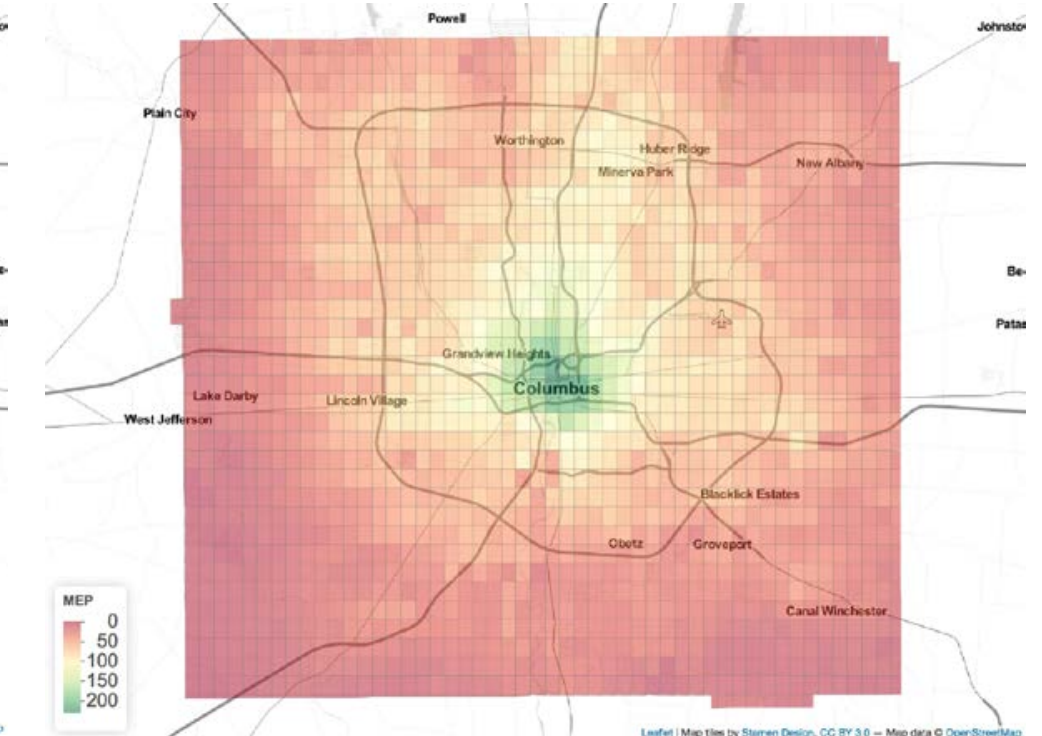

b)

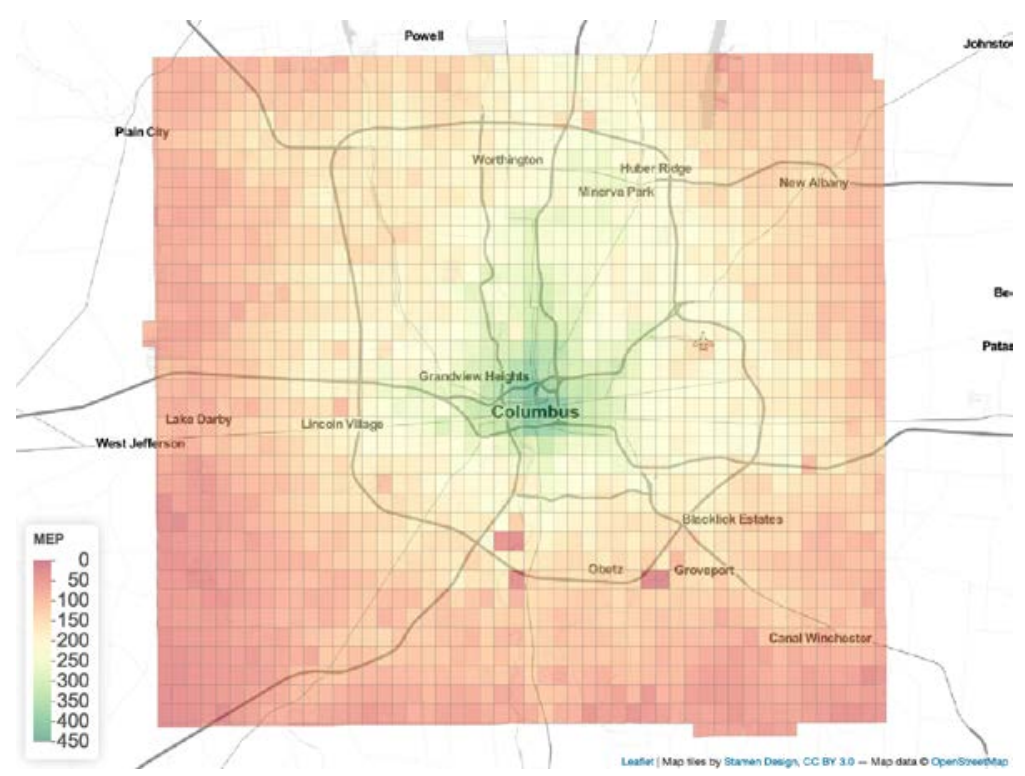

c)

FIGURE 4 MEP Maps for Columbus, Ohio, for: a) Car Mode; b) Walk, Bike, and Transit Modes (combined); c) All Modes 14

This report is available at no cost from the National Renewable Energy Laboratory (NREL) at www.nrel.gov/publications. 
In each of the figures, each square kilometer pixel is assigned a MEP metric. The figures are color coded using a red - green gradient scale where dark red depicts a score of zero (indicating low mobility energy productivity), and dark green indicates maximum MEP in the region (note that the scales are different in the different maps). Comparing the MEP metric of driving (FIGURE 4a) and all modes except driving (FIGURE 4b), it is evident that driving provides access to a greater number of opportunities than walk, bike, and transit combined. This finding goes in line with the saying, "most of our cities are built around automobile use." FIGURE 4c shows the overall MEP for Columbus (including driving walk, bike, and transit modes). Better MEP metrics are observed towards the city center where access to a variety of goods, services, and job opportunities are provided by energy-efficient modes when compared with suburban or exurban locations. Using a population proportion-based summation, the city-level MEP score for Columbus is 162 .

\section{SCENARIO ANALYSES}

Scenario analysis exercises were carried out to ensure that the MEP responds as anticipated to varying inputs. These are first-order analyses, meaning that the parameters for calculation of the MEP were changed to reflect anticipated primary impacts of hypothetical situations represented by each scenario.

For the first scenario analysis, a large increase in fuel economy (which could represent highpenetration of efficient vehicle technologies) was emulated in the Columbus region by adjusting car mode energy efficiency. To visualize MEP change in response to vastly increased vehicle fuel economy, the energy efficiency for automobiles was tripled compared to the baseline scenario for the MEP calculation (from 25 MPG to 75 MPG). Results of the scenario analysis are presented in FIGURE 5, reflecting more efficient mobility apart from the downtown (closer to the I-270 ring around Columbus) as would be expected from better fuel economy. These results verify one of the required properties of the $\mathrm{MEP}$ - with everything else remaining the same, if the energy efficiency of a mode increases, the overall MEP should increase accordingly.

A similar analysis was carried out to depict the impact of reduction in travel time (such as resulting from availability of faster travel modes). This was done by reducing the travel time deterrence of car mode, meaning that a distance that can be covered in 10 minutes in the baseline scenario can be covered in 3 minutes in the "faster travel mode" scenario. The results of this scenario analysis are presented in Figure 6. Once again, it can be observed that improving the travel efficiency of a mode improves the MEP scores not just along the downtown and freeway corridors, but in other places across the city.

These simplified scenario analyses capture only direct impacts to validate and check the formulation of the MEP. The scenarios do not take secondary or tertiary effects into account. Coupling the MEP with an agent-based travel microsimulation model could more fully capture the effects (primary and secondary) of new technologies such as induced/reduced demand or increased/decreased congestion. 


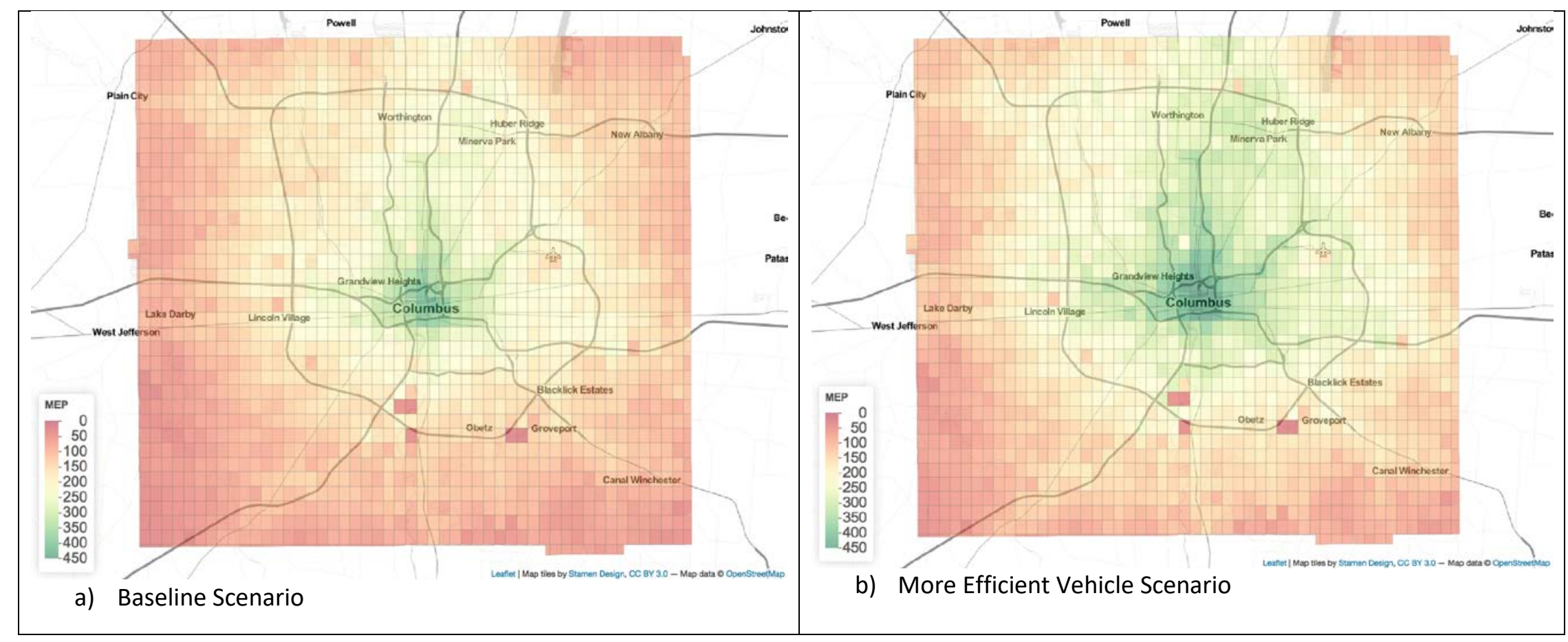

FIGURE 5. Impact of Vehicle Efficiency Improvement on MEP Scores 


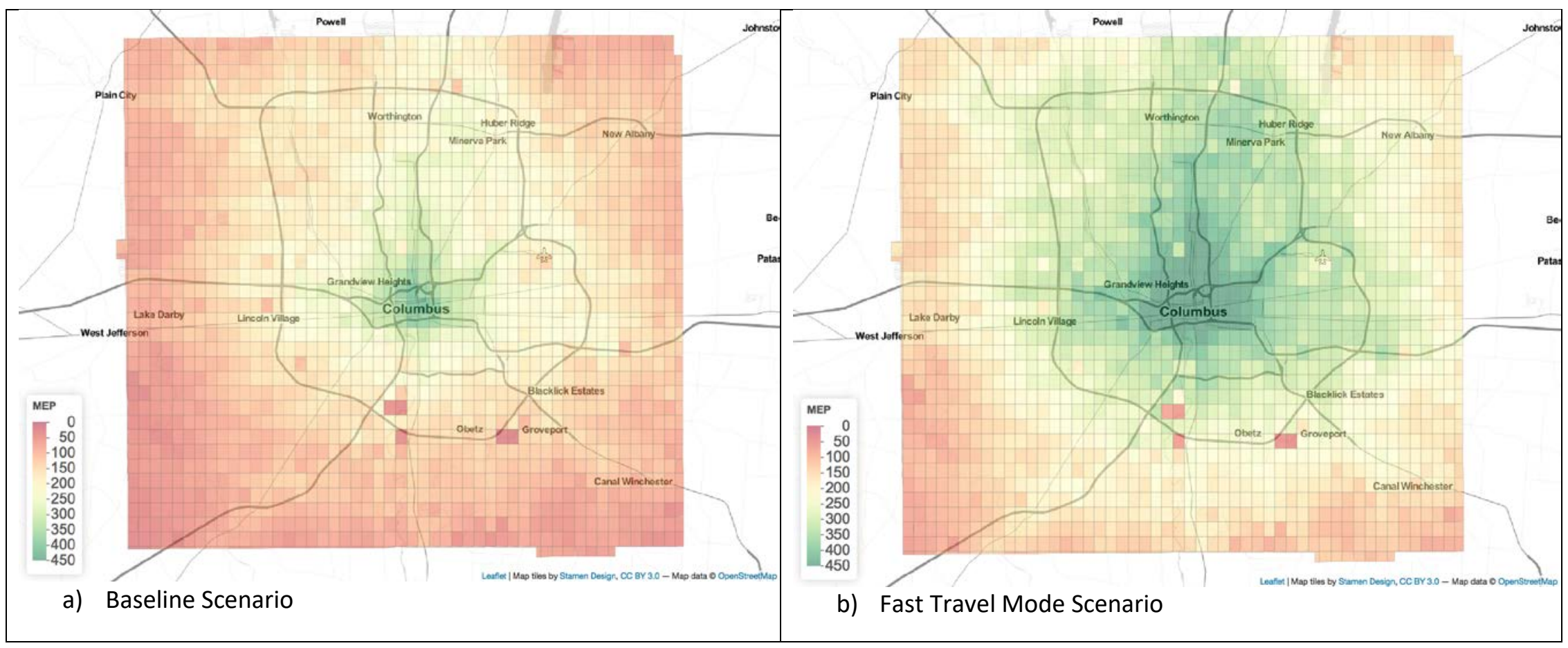

FIGURE 6. Impact of Faster Travel Mode on MEP Scores 


\section{CONCLUSIONS AND FUTURE WORK}

For the first time in many years (if not decades), passenger transportation and freight systems are experiencing significant innovation in moving increasing quantities of people and goods faster, cheaper, and with more choices and convenience. Be it the advent of shared mobility services or advancements in electric vehicle (EV) and automated vehicle (AV) technologies, the transportation field is undergoing a profound transformation, primarily fueled by technology and communications. All of these disruptive and transformative technologies will demand a suite of infrastructure investments and urban policy decisions that will redefine the way in which people view travel. While mobility technology has advanced, there remains a lack of comprehensive metrics that provide a holistic picture of the quality and energy efficiency of mobility options in a region. The MEP begins to address this gap, providing a quantitative lens to assess old and new mobility options moving forward. Building on concepts from accessibility theory (for which data availability and/or computational constraints have previously limited widespread application), the MEP provides a measure of access to goods, services, and employment, appropriately weighted by time, cost, and energy of modes that a person may use to reach these activities/opportunities.

This paper presents the MEP methodology and its implementation for the Columbus, Ohio, metropolitan region. The parameters and weighting coefficients used for aggregation across time, cost, energy, and activities were chosen from established literature and national data sets but can be customized based on region-specific knowledge. Furthermore, as an inherent place-based measure, the MEP can be spatially aggregated to measure mobility impacts at the neighborhood, district, city, or even national level while providing insights specific to a population sub-group, area, mode, or activity. The MEP is constructed such that data sources used for computation are readily available as well as easily adapted from urban travel demand models. Scenario analyses show that improvements in vehicle efficiency and travel times produced results consistent with desired metric attributes.

The MEP provides a useful lens for the DOE, planners, researchers, and metropolitan planning organizations to fairly assess the quality of mobility in a region, and to assess the impact of potential planning/investment strategies on regional mobility. When coupled with travel demand and/or regional land use models, the MEP can help assess not only current and emerging mobility technology, but also new travel modes and business models. The MEP methodology presented in this paper is a first attempt at developing a comprehensive measure of the quality of mobility of a region relative to energy efficiency. This initial work revealed a few limitations of the methodology that future research will address. In addition to travel time, energy, and travel cost (affordability), value of time experienced by individuals in different modal options (for example, the need to pay attention while driving a car, but flexibility to read a book or work on a laptop on a bus) needs to be taken into consideration in computing the MEP. The current MEP includes common modes such as car, transit, bike, and walk. Implementation needs to be extended to include new modes such as transportation networking companies (e.g., Uber, Lyft, and Via), laying the foundation to include shared automated mobility options. Future iterations of the metric will also incorporate mode-activity realism into the computation of the metric (to address unrealistic scenarios like walking 40 minutes to access a grocery store). Relevant data can be obtained from publicly available sources such as the National Household Travel Survey. Lastly, MEP measures need to be integrated with regional travel demand models to assess alternative future scenarios. Such integration will provide planners, researchers, and policy makers with a powerful tool to assess various investment strategies, policy decisions, and the consequences of new technologies. 


\section{AUTHOR CONTRIBUTION STATEMENT}

The authors confirm contribution to the paper as follows. Study conception and design: Venu Garikapati, Stan Young, and Yi Hou; Data collection: Yi Hou, Ambarish Nag, and Tom Grushka; Analysis and interpretation of results: Yi Hou, Venu Garikapati, Tom Grushka, and Ambarish Nag; Draft manuscript preparation: Venu Garikapati, Yi Hou, and Ambarish Nag. All authors reviewed the results and approved the final version of the manuscript.

\section{ACKNOWLEDGMENTS}

This work was authored by the National Renewable Energy Laboratory, operated by Alliance for Sustainable Energy, LLC, for the U.S. Department of Energy (DOE) under Contract No. DEAC36-08GO28308. Funding provided by the DOE Vehicle Technologies Office (VTO) under the Systems and Modeling for Accelerated Research in Transportation (SMART) Mobility Laboratory Consortium, an initiative of the Energy Efficient Mobility Systems (EEMS) Program. The authors acknowledge Stanley Young of NREL for leading the Urban Science Pillar of the SMART Mobility Laboratory Consortium. The authors would particularly like to thank David Anderson and Prasad Gupte with DOE's Office of Energy Efficiency and Renewable Energy (EERE) for helping to establish the project concept and advance its implementation, and for providing ongoing guidance and support. The views expressed in the article do not necessarily represent the views of the DOE or the U.S. Government. The U.S. Government retains and the publisher, by accepting the article for publication, acknowledges that the U.S. Government retains a nonexclusive, paidup, irrevocable, worldwide license to publish or reproduce the published form of this work, or allow others to do so, for U.S. Government purposes.

\section{REFERENCES}

1. Smith, A. Shared, Collaborative and On Demand: The New Digital Economy. http://www.pewinternet.org/2016/05/19/the-new-digital-economy/. Accessed November 2018.

2. Walker Consultants Reference. Ride-hailing Impacts on Parking. https://walkerconsultants.com/wp-content/uploads/2017/12/TNC-Impacts.pdf. Accessed November 2018.

3. Henao, A. Airport Analyses Informing New Mobility Shifts: Opportunities to Adapt EnergyEfficient Mobility Service and Infrastructure. Presented at the Intelligent Transportation Society of America Annual Meeting, 2018.

4. Ravenstein, E. G. The Laws of Migration. Journal of the Statistical Society of London, 1885. 48: $167-235$.

5. Silva, C. Comparative Accessibility for Mobility Management. The Structural Accessibility Layer. (Ph. D. Thesis). University of Porto, Porto, Portugal, 2008.

6. Busby, J. R. Accessibility-Based Transit Planning (M.S. Thesis). MIT, Cambridge, MA, 2004.

7. Warade, R. K. The Accessibility and Development Impacts of New Transit Infrastructure: The Circle Line in Chicago (M.S. Thesis). MIT, Cambridge, MA, 2007.

8. Ducas, C. R. Incorporating Livability Benefits into the Federal Transit Administration New Starts Project Evaluation Process through Accessibility-Based Modeling (M.S. Thesis). MIT, Cambridge, MA, 2011.

9. Peralta-Quirós, T., and Mehndiratta, S.R. Accessibility Analysis of Growth Patterns in Buenos Aires, Argentina: Density, Employment and Spatial Form. Transportation Research Record: Journal of the Transportation Research Board, 2015. 2512: 101-109.

10. Geurs, K. T, and van Wee, B. Accessibility Evaluation of Land-Use and Transport Strategies: Review and Research Directions. Journal of Transport Geography, 2004. 12: 127-140. 
11. Wachs, M. \& Kumagai, T. G. Physical Accessibility as a Social Indicator. Socioeconomic Planning Science, 1973, Vol. 7, No. 5, pp. 327-456.

12. Vickerman, R. W. Accessibility, Attraction and Potential: a Review of Some Concepts and Their Use in Determining Mobility. Environment and Planning A: Economy and Space, 1974, Vol. 6, No. 6, pp. 675-691.

13. Silva, C., and P. Pinho. The Structural Accessibility Layer (SAL): Revealing how Urban Structure Constrains Travel Choice. Environment and Planning A: Economy and Space, 2010, Vol. 42, No. 11, pp. 2735-2752.

14. Lee, M., and Holme, P. Relating Land Use and Human Intra-City Mobility. PLoS One, , 2015, Vol. 10, No. 10.

15. Noulas, A., Scellato, S.,Lambiotte, R., Pontil, M., and Mascolo, C. A Tale of Many Cities: Universal Patterns in Human Urban Mobility. , 2012, PLoS ONE, Vol. 7, No. 5.

16. Jung, W.-S., Wang, F., and Stanley, H. E. Gravity Model in the Korean Highway. Europhysics Letters, , 2008, Vol. 81, No. 4.

17. Krings, G., Calabrese, F., Ratti, C., and Blondel, V. D. Urban Gravity: A Model for Inter-City Telecommunication Flows. Journal of Statistical Mechanics: Theory and Experiment, 2009.

18. Levy, M. Scale-free Human Migration and the Geography of Social Networks. Physica A: Statistical Mechanics and its Applications, 2010, Vol. 389, No. 21, pp. 4913-4917.

19. Stouffer, S. A. Intervening opportunities: A Theory Relating Mobility and Distance.Am. Sociol. Rev., 1940, Vol. 5, No. 6, pp. 845-867.

20. Easa, S. M. Urban Trip Distribution in Practice. I: Conventional Analysis. Journal of Transportation Engineering, 1993, Vol. 119, No. 6, pp. 793-815.

21. Miller, E. A. Note on the Role of Distance in Migration: Costs of Mobility Versus Intervening Opportunities. Journal of Regional Science, 1972, Vol. 12, No. 3, pp. 475-478.

22. Haynes, K. E., Poston, D., and Sehnirring, P. Inter-Metropolitan Migration in High and Low Opportunity Areas: Indirect Tests of the Distance and Intervening Opportunities Hypotheses. Economic Geography, 1973, Vol 49, pp. 68-73.

23. Wadycki, W. J. Stouffer's Model of Migration: A Comparison of Interstate and Metropolitan Flows. Demography, 1975, Vol. 12, No. 1, pp. 121-128.

24. Cheung, C., and Black, J. Residential Location-Specific Travel Preferences in an Intervening Opportunities Model: Transport Assessment for Urban Release Areas. Journal of the Eastern Asia Society for Transportation Studies, 2005, Vol. 6, pp. 3773-3788.

25. Freymeyer, R. H., and Ritchey, P. N. Spatial Distribution of Opportunities and Magnitude of Migration. Sociological Perspectives, 2016, Vol. 28, No. 4, pp. 419-440.

26. Ben-Akiva, M. E., and Lerman, S. Discrete Choice Analysis. Cambridge, MA: MIT Press, 1985.

27. Dong, X. Analysis of Activity-Based Activity (M.S. Thesis). MIT, Cambridge, MA, 2000.

28. Dong, X., Ben-Akiva, M. E., Bowman, J. L., and Walker, J. L. Moving from Trip-Based to Activity-Based Measures of Accessibility. Transportation Research Part A, 2006, Vol. 40, No. 2, pp. 163-180.

29. Simini, F., González, M. C., Maritan, A., and Barabási, A.-L. A Universal Model for Mobility and Migration Patterns. Nature, 2012, Vol. 484, No. 7392, pp. 96-100.

30. Walk Score ${ }^{\circledR}$. Live Where You Love. https://www.walkscore.com/. Accessed July 2018.

31. TransitScreen ${ }^{\circledR}$. TransitScreen. https://transitscreen.com/mobilityscore/. Accessed July 2018. 
32. Rendall, S., Page, S., Reitsma, F., Van Houten, E., and Krumdieck, S. Quantifying Transport Energy Resilience. Transportation Research Record: Journal of Transportation Research Board, 2011, Vol. 2242, pp. 72-80.

33. Inturri, G., Ignaccolo, M., Le Pira, M., Capri, S., and Giuffrida, N. Influence of Accessibility, Land Use and Transport Policies on Transport Energy Dependence of a City. Transportation Research Procedia, 2017, Vol. 25, pp. 3273-3285.

34. Saunders, M. J., Kuhnimhof, T., Chlond, B., and Rodrigues da Silva, A. N. Incorporating Transport Energy into Urban Planning. Transportation Research Part A: Policy and Practice, 2008, Vol. 42, No. 6, pp. 874-882.

35. Karathodorou, N., Graham, D. J., and Noland, R. B. Estimating the Effect of Urban Density on Fuel Demand. Energy Economics, 2010, Vol. 32, No. 1, pp. 86-92.

36. Nag, A., Hou, Y., Garikapati, V.M., and Young, S. Literature Review on Accessibility Metrics. Working Paper: National Renewable Energy Laboratory, 2018.

37. Owen, A., and Levinson, D. M. Access Across America: Transit 2014. Minneapolis, MN: Center for Transportatio Studies, Univeristy of Minnesota, 2014.

38. Mapzen. https://mapzen.com. Accessed July 2018.

39. OpenStreetMap. https://www.openstreetmap.org. Accessed July 2018.

40. Transitland data. https://transit.land. Accessed July 2018.

41. General Transit Feed Specification https://www.transitwiki.org/TransitWiki/index.php/General_Transit_Feed_Specification. Accessed July 2018.

42. Costar. http://www.costar.com. Accessed July 2018.

43. Google Places. Retrieved from: https://developers.google.com/places/web-service/intro

44. FourSquare. Retrieved from: https://foursquare.com

45. Longitudinal Household Employer Dynamics (LEHD) Origin-Destination Employment Statistics of the U.S. Census. Retrieved from: https://lehd.ces.census.gov/data/

46. Oak Ridge National Laboratory. Transportation Energy Data Book. https://cta.ornl.gov/data/index.shtml. Accessed November 2018.

47. Federal Transit Administration. National Transit Summary \& Trends, 2015.

48. American Automobile Association. Your Driving Costs. https://newsroom.aaa.com/auto/yourdriving-costs/. Accessed November 2018.

49. National Household Travel Survey. Retrieved from: https://nhts.ornl.gov/

50. U.S. Census 2016 American Community Survey. Retrieved from: https://www.census.gov/programs-surveys/acs/ 